\title{
NOTAS
}

\section{DISTRIBUCIÓN DEMOGRAFICA DE LA ASIBILACIÓN DE VIBRANTES EN EL HABLA DE LA CIUDAD DE MÉXICO}

El estudio de la distribución de variables fonéticas en una zona urbana sociológicamente compleja se basa en el supuesto de que no todas las variantes fonéticas se encuentran con la misma frecuencia en el inventario fonético de todos los hablantes, y que, en algunos casos, pueden faltar completamente. El presente estudio se propone analizar la frecuencia de la asibilación de las vibrantes en el español hablado en la ciudad de México, con objeto de puntualizar el sector de la población que es, o ha sido, el foco de irradiación del fenómeno en cuestión. Se espera así proyectar diacrónicamente un hecho lingüístico sincrónico ${ }^{~}$.

El material para este estudio consiste en 110 horas de conversaciones libres entre un informante y un investigador que, en forma de cintas magnetofónicas, se guardan en el Centro de Estudios Lingüísticos y Literarios de El Colegio de México. Tales grabaciones fueron realizadas por investigadores de dicho Centro entre los años de 1963 y 1969. La uniformidad del método de encuesta queda garantizada por la uniformidad de la preparación académica de los investigadores.

La naturaleza de este trabajo obligó a que cada informante satisficiera ciertos requisitos: a) que hubiera nacido en la ciudad de México o que hubiera vivido en ella gran parte de su vida; $b$ ) que sus padres fueran de habla española y de preferencia mexicanos; $c$ ) que hubiera hecho sus estudios en México. Se clasificó a cada informante, además, según el sexo, el grupo generacional y la clase socioeconómica. Mientras que la clasificación según el sexo no necesita mayor explicación, el establecer grupos generacionales presentó problemas que se resolvieron con mira al objetivo de este trabajo. Puesto que cualquier segmentación de la población tiene que ser arbitraria, la que aquí se propone, en tres gru pos generacionales, no quiere ser sino una de tantas posibles: el GG I, de los 16 a los 32 años de edad; el GG II, de 33 a 55; y el GG III, de 56 en adelante. Fueron excluidos hablantes menores de 16

1 Como modelo de análisis sociolingüístico de una zona urbana proponemos el libro de Wincram Labov, The social stratification of English in New York City, New York, 1966. 
años porque se consideró que su habla estaría bajo la influencia normativa de los maestros $y$, por consiguiente, no representaría un sector autóctono específico. El Grupo Generacional I quiere reflejar el habla del segmento de la población comúnmente llamado "la juventud", que se encuentra en una etapa de autorrealización y que tiene conciencia de su identidad como grupo aparte. El GG II se compone de individuos que se caracterizan por haber alcanzado la madurez y estabilidad, tanto emocional como profesional, y que también muestran voluntad de formar un grupo aparte. Los informantes del GG III se consideran, por lo general, miembros de una sociedad que ya no existe y portadores de valores que muchos ya rechazan; sus hábitos están ya formados desde hace mucho y se resisten tenazmente a cualquier cambio ${ }^{2}$.

La división de la población en grupos socioeconómicos presentó también problemas complejos que se resolvieron pensando en la naturaleza preeminentemente lingüística de este estudio. No se pretende aquí diferenciar clases sociales según sus hábitos lingüísticos, sino más bien ensayar un método que permita identificar el foco de irradiación de un fenómeno partiendo del análisis del habla de cierto grupo de hablantes. Se adoptaron cuatro criterios para clasificar al informante: $a$ ) educación (primaria, secundaria, postsecundaria) ; b) prófesión; c) condición económica; d) conciencia del mundo que lo rodea. Se llegó así a la constitución de tres grupos socioeconómicos. El GSE 3 se compone de personas que por lo menos han'terminado los estudios secundarios, poseen bienes materiales, tienen cierto prestigio social, ocupan altos cargos en su profesión, son intelectuales y a menudo muestran actitudes cosmopolitas. El GSE 2 está compuesto por lo general de individuos con educación secundaria, que tienen que trabajar para poder sobrevivir o son dueños de pequeños negocios, constantemente emulan a la clase alta y son conscientes sólo de los acontecimientos a nivel nacional. El GSE 1 se compone de personas que casi no han recibido instrucción formal, viven de un trabajo casi siempre manual, apenas sobreviven de día en día, y casi no tienen conciencia de lo que no experimentan personalmente ${ }^{3}$.

Asibilación de La vibrante sIMPLe /r/

La existencia de una variante fricativa asibilada $[r]^{4}$ ha sido documentada para varios lugares de Hispanoamérica: Nuevo México, Costa

2 Esta segmentación no difiere de manera significativa de las de Labov, op. ctt., de Anshen (infra, nota 8) y de Juan M. Lope Blangh, La filologia hispánica en México, México, 1969, p. 49.

3 Para las clases sociales de México véase el volumen colectivo intitulado Ensayo sobre las clases sociales en México, México, 1968, especialmente los artículos de Pablo GonzÁlez Casanova, "Enajenación y conciencia de clases en México", pp. 152-194, y de Nathan L. Whetten, "El surgimiento de una clase media en México", pp. 63.94; también Lucio Mendieta y NúÑEZ, Las clases sociales, México, 1947. Para un planteamiento reciente de los problemas sociolingüusticos, véase ÓsCar URIBE Villegas, Sociolingüistica, México, 1970.

* Las limitaciones de la imprenta nos obligan a prescindir de signos fonéticos. 
Rica, Guatemala, Colombia, Sierra del Ecuador, Paraguay, Chile, Argentina, y México ${ }^{5}$. Cabe sospechar que este fenómeno es de formación reciente en México, ya que la tesis de Marden no lo menciona, y Matluck, en 1951, registra sólo casos esporádicos de $[\mathrm{t} r],[\mathrm{d} r]^{6}$. Mi propia investigación, sin embargo, revela que la asibilación de la vibrante simple se da con frecuencias muy altas en el habla de todos los sectores de la población $y$, además, que estas frecuencias varían considerablemente según el sector que se analice. Para cuantificar las frecuencias de $[r]$, he tomado en cuenta únicamente la posición final ante pausa y he dividido todas las realizaciones de $/ \mathrm{r} /$ en dos categorías: a) $[r]$ asibilada, que incluye $[r r]$ y las variantes ensordecidas de $[r]$ y $[r r] ; b)[r]$ no asibilada, que incluye $[\mathrm{rr}]$ y las variantes fricativas de $[\mathrm{r}]$ y $[\mathrm{rr}]$. Un primer cómputo de las apariciones de $/ \mathrm{r} /$ da los siguientes resultados:

$\begin{array}{llr}{[\mathrm{r}]} & 276 & 31.8 \% \\ {[r]} & 560 & 68.2 \% \\ \text { TOTAL } & 836 & 100.0 \%\end{array}$

La frecuencia de la variante asibilada es tan alta que parece contradecir cualquier observación impresionista que se pueda hacer in situ. Es necesario, pues, analizar los datos según un primer eje posible: el sexo del informante. Se obtiene así la siguiente tabla:

\begin{tabular}{llll} 
& \multicolumn{1}{c}{$[\mathrm{r}]$} & \multicolumn{1}{c}{$[r]$} & Total \\
MUJERES & $18.2 \%$ & $81.8 \%$ & $100.0 \%$ \\
& 100 & 448 & 548 \\
HoMbres & $61.1 \%$ & $38.9 \%$ & $100.0 \%$ \\
& 176 & 112 & 288 \\
Total & 276 & 560 & 836
\end{tabular}

En vista de que la asibilación de la vibrante parece ser un fenómeno reciente, los porcentajes obtenidos no dejan de causar sorpresa, ya que es tradicional considerar a la mujer lingüísticamente conservadora ${ }^{7}$. El cómputo por sexos afina la frecuencia global: las mujeres asibilan la $/ \mathbf{r} /$ mucho más que los hombres.

Indicamos con $[\mathrm{r}]$ la vibrante simple y con $[\mathrm{rr}]$ la vibrante múltiple, y con $[r]$ y $[r r]$, respectivamente, sus variantes asibiladas.

5 DANIEL N. CÁRdenas, "The geographic distribution of assibilated $R, R R$ in Spanish America", Orbis, 7 (1958), 407-414.

"Charles Marden, "La fonología del español en la ciudad de México", $B D H, 4$ (1938) , 87-187; en realidad los datos son de la tesis de doctorado que presentó Marden en la Universidad de Johns Hopkins en 1896; JOSEPH MatLuCK, "La pronunciación del español en el Valle de México", NRFH, 6 (1952), 102-120.

7 Véase, por ejemplo, el estudio de Gregorio Salvador, "Fonética masculina y fonética femenina en el habla de Vertientes y Tarifa (Granada)", Orbis, 1 (1952), 19-24; casi todo el primer tomo de Orbis trata del habla de las mujeres en distintas partes del mundo. 
Ahora bien, la distribución de esta variable también es cuantificable según ejes generacionales (GG) :

\begin{tabular}{llll} 
& \multicolumn{1}{c}{$[\mathrm{r}]$} & \multicolumn{1}{c}{$[r]$} & ToraL \\
GG I & $26.5 \%$ & $73.5 \%$ & $100.0 \%$ \\
& 124 & 344 & 468 \\
GG II & $35.5 \%$ & $64.5 \%$ & $100.0 \%$ \\
& 108 & 196 & 504 \\
GG III & $68.7 \%$ & $31.3 \%$ & $100.0 \%$ \\
& 44 & 20 & 64 \\
TotaL & 276 & 560 & 836
\end{tabular}

Si la hipótesis de que la variante asibilada es un fenómeno reciente se basaba únicamente en la falta de documentación anterior, los porcentajes obtenidos según la edad de los hablantes permiten examinarla bajo una nueva perspectiva. La $[r]$ es mucho más común entre los hablantes de los GG I y II que entre los del GG III, lo que nos hace suponer que la baja frecuencia entre los mayores de edad significa desarrollo tardio o, con más precisión, llevado a cabo durante los últimos 30 o 40 años ${ }^{8}$. Se podría sospechar, sin embargo, que la alta frecuencia de la asibilada en los Grupos Generacionales I y II no es sino reflejo del eje anterior, el masculino-femenino. Para rechazar este argumento es necesario analizar los mismos datos tomando en cuenta tanto el sexo como la edad del informante. Simplifico la presentación y doy a conocer únicamente los porcentajes:

$\begin{array}{lccc} & {[r]} & {[r]} & \text { Total } \\ \text { MUJERES } & & & \\ \text { GG I } & 80.0 \% & 20.0 \% & 100.0 \% \\ \text { GG II } & 87.5 \% & 12.5 \% & 100.0 \% \\ \text { GG III } & 71.5 \% & 28.5 \% & 100.0 \% \\ \text { HoMBRES } & & & \\ \text { GG I } & 50.0 \% & 50.0 \% & 100.0 \% \\ \text { GG II } & 34.0 \% & 65.0 \% & 100.0 \% \\ \text { GG III } & 0.0 \% & 100.0 \% & 100.0 \%\end{array}$

Este análisis simultáneo pone de relieve que los dos sexos se diferencian aún más en el uso de la variante asibilada si se distribuyen en grupos generacionales. Entre las mujeres, la $[r]$ es frecuente aun en personas de edad, lo que podría llevar a la conclusión de que no se trata de desarro-

8 Frank ANshen, "A sociolinguistic analysis of a sound change", Language Sciences, núm. 9 (febrero de 1970), 20-21, aplica el mismo método y obtiene resultados similares para la variable / $\mathrm{r}$ / postrocálica inglesa en el habla de una comunidad negra en el Sur de los Estados Unidos. 
llo tardío. Un análisis detallado de los datos, sin embargo, subraya que la diferencia en el uso de la asibilada entre el GG I y el GG III es sólo del $8.5 \%$, y que no aumenta progresivamente, sino que en el GG II hay mayor frecuencia de $[r]$ que en el GG I. Entre los hombres, por el contrario, la asibilada disminuye, hasta la ausencia total en el GG III, conforme aumenta la edad del informante.

Si se analizan los datos según los tres grupos socioeconómicos mencionados arriba, se obtiene el siguiente cuadro:

\begin{tabular}{llll} 
& \multicolumn{1}{c}{$[r]$} & \multicolumn{1}{c}{$[\mathrm{r}]$} & TotaL \\
GSE 1 & $53.9 \%$ & $46.1 \%$ & $100.0 \%$ \\
& 84 & 72 & 156 \\
GSE 2 & $80.8 \%$ & $19.2 \%$ & $100.0 \%$ \\
& 268 & 64 & 332 \\
GSE 3 & $59.8 \%$ & $40.2 \%$ & $100.0 \%$ \\
& $208 \%$ & 140 & 348 \\
TotaL & 560 & 276 & 836
\end{tabular}

Lo que parece desprenderse de estos porcentajes es la falta de una estratificación marcada para el GSE 1 y el GSE $3 \mathrm{y}$, sorprendentemente, la clarísima preferencia por la variante asibilada entre los informantes pertenecientes al GSE 2. Aunque estas consideraciones son válidas en líneas generales, es preciso otra vez estudiar el fenómeno entrecruzando el eje socioeconómico con el del sexo de los hablantes, a fin de averiguar si tan altos porcentajes se-deben al sexo. Se obtienen así los siguientes datos:

$$
[r] \quad[r] \quad \text { Total }
$$

Mujeres

$\begin{array}{llll}\text { GSE 1 } & 79.4 \% & 20.6 \% & 100.0 \% \\ \text { GSE 2 } & 87.1 \% & 12.9 \% & 100.0 \% \\ \text { GSE 3 } & 86.0 \% & 14.0 \% & 100.0 \%\end{array}$

HOMBres

$\begin{array}{llll}\text { GSE 1 } & 20.0 \% & 80.0 \% & 100.0 \% \\ \text { GSE 2 } & 59.0 \% & 41.0 \% & 100.0 \% \\ \text { GSE 3 } & 36.1 \% & 63.9 \% & 100.0 \%\end{array}$

De nuevo notamos que las mujeres se distinguen de los hombres en el tratamiento de la $/ \mathrm{r} /$ en posición final absoluta, aun si se toma en cuenta la clase socioeconómica del hablante. Las mujeres invariablemente exhiben una mayor frecuencia de $[r]$ en todos los GSE. Entre los hombres, por el contrario, la variante no asibilada es la más común, excepto para el GSE 2, que tiene un porcentaje notablemente alto de $[r]$ $(59.0 \%)$, pero todavía lejos del más bajo entre las mujeres $(79.4 \%$ para 
el GSE 1). Si bien el sexo del hablante es el que determina en gran medida el porcentaje de una $u$ otra variante, no es menos cierto que aun así las clases bajas de ambos sexos tienen menor frecuencia de $[r]$ que las clases media y alta. Esto parece reforzar la hipótesis según la cual los estratos inferiores de la población son más resistentes al cambio y conservan formas lingüísticas en vías de desuso entre los demás sectores.

En conclusión, el análisis detallado de la distribución de las variantes asibiladas del fonema / $\mathbf{r}$ / confirma la sospecha no documentable de que $[r]$ es de desarrollo reciente. Notamos, de hecho, que ésta es más frecuente entre los jóvenes que entre los mayores. Vimos, además, que las clases media y alta asibilan la vibrante simple con mucha mayor frecuencia que la baja, aunque ésta ciertamente no desconoce el fenómeno. Por último, la nueva articulación asibilada es mucho más favorecida por las mujeres que por los hombres, y no ha de descartarse la idea de que se haya originado entre ellas. Así, pues, podemos afirmar que los centros de difusión de la variante $[r]$ son las mujeres, los jóvenes y las clases media y alta.

\section{Asibilación de la vibRante Múltiple / rr /}

Mientras que la vibrante simple se asibila únicamente en posición final absoluta y en el grupo / tr/, la vibrante múltiple puede asibilarse en cualquier contorno fonético. Igual que en el caso de la simple, dividimos todas las realizaciones de / rr/ en dos grupos: a) [rr] asibilada, que incluye una variante ensordecida, y $b$ ) $[\mathrm{rr}]$ no asibilada, que incluye una variante fricativa. Un primer cómputo proporciona los siguientes porcentajes:

$\begin{array}{lrr}{[r r]} & 496 & 31.5 \% \\ {[\mathrm{rr}]} & 1076 & 68.5 \% \\ \text { Total } & 1572 & 100.0 \%\end{array}$

El hecho de que la variante asibilada de la vibrante múltiple tenga una frecuencia mucho menor que la de la simple se explica, claro está, por el hecho de que en esta sección se estudian las apariciones de / $r$ r / en cualquier contexto, mientras que para $/ \mathrm{r} /$ nos limitamos a analizar la posición final absoluta. Aun así, no deja de sorprender la alta frecuencia de la asibilada larga (que ya no múltiple). Analicemos ahora los datos según el sexo de los informantes:

\begin{tabular}{llll} 
& \multicolumn{1}{c}{$[r r]$} & \multicolumn{1}{c}{$[\mathrm{rr}]$} & Total \\
MUJERES & $38.5 \%$ & $61.5 \%$ & $100.0 \%$ \\
& $364 \%$ & 580 & 944 \\
HOMBRES & $21.0 \%$ & $79.0 \%$ & $100.0 \%$ \\
& 132 & 496 & 628 \\
Total & 496 & 1076 & 1572
\end{tabular}


Sin olvidar lo que acabamos de afirmar, la variante no asibilada es considerablemente más frecuente que la asibilada, pero, igual que en el caso del fonema estudiado antes, las mujeres asibilan con mucha mayor frecuencia que los hombres.

El análisis según grupos generacionales se puede resumir en la siguiente tabla:

\begin{tabular}{llll} 
& \multicolumn{1}{c}{$[r r]$} & \multicolumn{1}{c}{$[\mathrm{rr}]$} & \multicolumn{1}{c}{ TotaL } \\
GG I & $35.8 \%$ & $64.2 \%$ & $100.0 \%$ \\
& 340 & 608 & 948 \\
GG II & $34.5 \%$ & $65.5 \%$ & $100.0 \%$ \\
& 156 & 296 & 492 \\
GG III & $0.0 \%$ & $100.0 \%$ & $100.0 \%$ \\
& 0 & 172 & 172 \\
TotaL & 496 & 1076 & 1572
\end{tabular}

La falta total de la variante asibilada para los hablantes del Grupo Generacional III confirma lo que se propuso para la asibilación de la vibrante simple: que es un fenómeno de formación reciente en el español de la ciudad de México. Más aún, la aparición del fenómeno debió de ser repentina, puesto que la frecuencia aumenta espectacularmente en el GG II y es casi igual a la del GG I. En vista de esto último y de la diferenciación entre los dos sexos en el tratamiento de $/ \mathrm{rr} /$, se presenta a continuación el análisis por sexo y grupo generacional:

$$
[r r] \quad[\mathrm{rr}] \quad \text { Total }
$$

MUJERES

$\begin{array}{lrrr}\text { GG I } & 41.8 \% & 58.2 \% & 100.0 \% \\ \text { GG II } & 48.6 \% & 51.4 \% & 100.0 \% \\ \text { GG III } & 0.0 \% & 100.0 \% & 100.0 \%\end{array}$

Hombres

$\begin{array}{lrrr}\text { GG I } & 20.3 \% & 79.7 \% & 100.0 \% \\ \text { GG II } & 27.6 \% & 72.4 \% & 100.0 \% \\ \text { GG III } & 0.0 \% & 100.0 \% & 100.0 \%\end{array}$

Mientras que la tabla anterior no permitía llegar a diferenciar el GG I del GG II, debido a que ambos grupos exhibian casi la misma frecuencia de la variante asibilada, el análisis simultáneo de dos ejes revela que sí hay diferencias entre estos dos grupos en el tratamiento de /rr/; concretamente, el GG II asibila más que el GG I, y esto de manera constante para los dos sexos (con una diferencia del $7 \%$ en cada uno de ellos). Esta tabla confirma también lo que una y otra vez hemos venido observando: que la alta frecuencia de las variantes asibiladas es en realidad función de su alto porcentaje entre las mujeres. Prueba de 
ello es el hecho de que si comparamos los porcentajes de los hombres en esta última tabla con los obtenidos anteriormente en el eje hombresmujeres, salta a la vista que son casi idénticos, lo que significa que, en lo que a la asibilación se refiere, es necesario tener en cuenta siempre, como lo hemos hecho, el sexo del informante.

El análisis de la variable en cuestión según grupos socioeconómicos se resume en la siguiente tabla:

\begin{tabular}{llll} 
& \multicolumn{1}{c}{$[\mathrm{rr}]$} & \multicolumn{1}{c}{$[\mathrm{rr}]$} & Total \\
GSE 1 & $17.7 \%$ & $82.3 \%$ & $100.0 \%$ \\
& 56 & 260 & 316 \\
GSE 2 & $39.7 \%$ & $60.3 \%$ & $100.0 \%$ \\
& 256 & 388 & 644 \\
GSE 3 & $30.0 \%$ & $70.0 \%$ & $100.0 \%$ \\
& 184 & 428 & 612 \\
Total & 496 & 1076 & 1572
\end{tabular}

Está claro que hay estratificación social de esta variable, puesto que existe una diferencia notable de frecuencias entre la clase baja y las dos superiores. Las clases media y alta asibilan con mucha mayor frecuencia que la baja, hasta el punto de que podría afirmarse que sólo hay dos grupos socioeconómicos para esta variable o, mejor dicho, que sólo hay dos grupos sociolingüísticamente pertinentes para la asibilación de la vibrante múltiple. Pero nuevamente es preciso analizar los datos entrecruzando los ejes:

$$
[r r] \quad[\mathrm{rr}] \quad \text { TotaL }
$$

Mujeres

$\begin{array}{llll}\text { GSE 1 } & 28.5 \% & 71.5 \% & 100.0 \% \\ \text { GSE 2 } & 40.3 \% & 59.7 \% & 100.0 \% \\ \text { GSE 3 } & 38.0 \% & 62.0 \% & 100.0 \%\end{array}$

HOMBRES

$\begin{array}{llll}\text { GSE 1 } & 13.9 \% & 86.1 \% & 100.0 \% \\ \text { GSE 2 } & 38.2 \% & 61.8 \% & 100.0 \% \\ \text { GSE 3 } & 18.0 \% & 82.0 \% & 100.0 \%\end{array}$

Estos porcentajes subrayan una vez más la importancia del eje hombres-mujeres para la pronunciación $[r r]$. Los hombres del GSE 1 tienen una frecuencia bastante baja $(13.9 \%)$ de la asibilada, y las mujeres del mismo grupo los superan de manera considerable (28.5\%). En el GSE 2, sin embargo, el sexo del informante no parece ser pertinente para la realización fonética del fonema $/ \mathrm{rr} /$, así que los dos sexos tienen frecuencias muy similares $(38.2 \%$ y $40.3 \%)$. Es en la clase superior donde la diferencia de sexo se vuelve distintiva: las mujeres del GSE 3 tienen una frecuencia de $[r r]$ muy superior a la de los hombres, cuyo porcentaje se acerca aquí al de la clase baja. 
En conclusión se puede afirmar que en la ciudad de México la vibrante múltiple se asibila menos que la vibrante simple, pero, claro está, mientras que ésta se asibila casi exclusivamente en posición final absoIuta, aquélla puede realizarse como $[\mathrm{rr}]$ en cualquier posición. Sobre esto se podría argumentar que debido a que la combinación /s/ + /rr favorece la asibilación, en los demás contextos se trataría de una extensión de un hábito articulatorio preexistente. En realidad, de los 496 casos de $[r r], 168$, o sea $33.8 \%$, son de $/ \mathrm{s} /+/ \mathrm{rr} /$, porcentaje que no creo suficientemente alto para justificar tal hipótesis. Quizá sería más aceptable proponer que, dada la combinación $/ \mathrm{s} /+/ \mathrm{rr} /$, las probabilidades de $[r r]$ son altas. En efecto, de 308 casos de $/ \mathrm{s} /+/ \mathrm{rr} /, 168$, o sea $54.8 \%$, se pronunciaron con la asibilada, que, vista así, es articulación mayoritaria.

Aun así, sin embargo, la variante vibrante es más común que la asibilada. Pero en vista de que ésta es más común para la $/ r /$ simple, no ha de descartase la posibilidad de que en un futuro más o menos cercano la vibrante pase a ser una articulación minoritaria destinada a desaparecer. Notamos, en efecto, que la $[r r]$ es común entre mujeres de cualquier grupo y entre algunos hombres. Cabe subrayar, además, que la asibilación es socialmente aceptada por la clase alta y, por consiguiente, común entre la media. Con estas consideraciones creo que se puede proyectar la asibilada hacia el futuro y afirmar que irá adquiriendo más popularidad debido al impulso recibido por las mujeres de todas las edades y grupos socioeconómicos, por la clase media de ambos sexos, y con el consentimiento tácito de los hombres de la clase alta.

State University of New York

Giorgio Perissinotto at Stony Brook

\section{UN RASGO ESTILISTICO DEL ROMANGERO Y DE LA LÍRICA POPULAR}

Si hojeamos un moderno cancionero de lírica popular, encontraremos muchas coplas enumerativas construidas sobre, el siguiente esquema: una cuarteta en que se contienen tres elementos, los dos primeros desarrollados cada uno en un verso y el último en dos:

\footnotetext{
Para San Juan son las rosas, para San Pedro los ramos, pa el bendito San Antonio los claveles encarnados.
}

(Burgos: GIL-II, p. 52) ${ }^{1}$.

1 He aquí las abreviaturas empleadas:

Catalán: Diego Catalán, La flor de la marañuela. Romancero general de las islas Canarias, 2 ts., Madrid, 1969.

Córdova: Sixto Córdova y OÑa, Cancionero popular de la provincia de Santander, 4 ts., Santander, 1947. 